\title{
Retinoblastoma treatment outcomes in Pakistan.
}

1. FRCS, FCPS, FPO

Assistant Professor Peadiatric

Ophthalmology

The Children's Hospital \& Institute of Asma Mushtaq ${ }^{1}$, Asad Aslam Khan², Mohammad Ali Ayaz Sadiq ${ }^{3}$, Seema Qayyum ${ }^{4}$, Child Health, Lahore.

2. MS, PhD

Professor Ophthalmology KEMU/Mayo Hospital, Lahore.

3. FCPS, FAO

Associate Professor Ophthalmology KEMU/Mayo Hospital, Lahore.

4. FCPS

Professor Ophthalmology

The Children's Hospital \& institute of Child Health, Lahore.

5. FCPS

Senior Registrar Ophthalmology Holy family Hospital, Rawalpindi.

6. FCPS, MRCS

Senior Registrar Ophthalmology The Children's Hospital \& institute of Child Health, Lahore.

Correspondence Address:

Dr. Asma Mushtaq

House No. 253, D1, Nespak Society,

Lahore.

drasmamushtaq18@gmail.com Muhammad Rizwan Khan ${ }^{5}$, Ahmed Raza ${ }^{6}$

ABSTRACT... Objective: To compare regression of retinoblastoma lesion after monotherapy (chemotherapy) vs combined therapy (chemotherapy + local consolidative therapy). Study Design: Randomized Control trial. Setting: Department of Pediatric Ophthalmology at College of Ophthalmology and Allied Vision Sciences/ King Edward Medical University, Mayo Hospital Lahore, Pakistan. Period: January 2015 to June 2016. Material \& Methods: 43 patients with retinoblastoma (group C \& D) were included in the study. Chemotherapy was given to patients in group1 and chemotherapy followed by local consolidative therapy was given to patients in group2. The percentage of regression of baseline lesion was noted after 6 cycles of chemotherapy. Results: The mean regression of lesion in group 1 was $24.5 \%$ and in group2 was $59.9 \%$, after six months of treatment. The value t-test was 36.02 with p value $0.00(<0.05)$. Conclusion: The combined modality of chemotherapy and local consolidative therapy is more effective in causing regression of retinoblastoma lesion than chemotherapy.

Article received on:

Key words: $\quad$ Chemotherapy, Laser, Retinoblastoma, Tumor, Treatment.

08/02/2020

Accepted for publication:

13/04/2020

\section{INTRODUCTION}

Retinoblastoma having an incidence of 1 in 15,000 has the most common presentation as leukocoria and strabismus. ${ }^{1}$ Its management is highly individualized requiring a multidisciplinary approach with the aim of saving the life of the patient, followed by globe and any useful vision if possible. ${ }^{2}$

In the last century, a paradigm shift is seen in the management of retinoblastoma. Studies completed by the Retinoblastoma Study Group show promising use of chemotherapy as a primary mode of treatment in reducing tumor bulk, followed by various forms of local approaches that can be used for final tumor control. According to Canadian National strategy guidelines, Group A is treated by focal lasers alone, Group B, C \& $D$ treated by combined chemotherapy and focal lasers. Enucleation is mainly reserved for group E tumors and group D tumors not responding to chemotherapy. ${ }^{1,3}$
A study from London reported that the fiveyear survival rate for children with unilateral retinoblastoma increased from $85 \%$ in $20^{\text {th }}$ century to $97 \%$ in $21{ }^{\text {st }}$ century. ${ }^{4}$ A study conducted by Teming in Germany showed overall survival rate of $95 \%$ each year. ${ }^{5}$ However, reports from Africa predict a disease-free survival of around $20 \% .{ }^{6}$ Kivelia in his editorial highlighting the advancement in the medical field in last century, concluded that, it may take another 200 years for the benefit to spread worldwide. ${ }^{7}$

According to the best of our knowledge, no study has been done in Pakistan to compare the treatment outcomes of Retinoblastoma patients. Studies from Pakistan highlight epidemiology and their clinic presentations..$^{8-11}$ The objective of our study was to utilize the latest treatment guidelines to save the life of the patient as well as prevent organ loss as enucleation and external beam radiotherapy has largely been abandoned by the developed world..$^{8-10}$ 


\section{MATERIAL \& METHODS}

This prospective randomized control trial was conducted in the department of Pediatric Ophthalmology at College of Ophthalmology and Allied Vision Sciences/ King Edward Medical University, Mayo Hospital Lahore between January 2015 to June 2016. The study was approved by the Institutional Review Board of King Edward Medical University Lahore. An informed consent was obtained from the parents of patients prior to inclusion in the study.

88 patients with retinoblastoma presenting to the Outpatient clinic were screened after taking an informed consent. Patients who had had previous treatment or were not suitable for chemotherapy were excluded from the study. 43 patients having Group C or D lesion were included in the study. They were randomly divided into two groups with Group 1 patients undergoing 6 cycles of Intravenous chemotherapy (vincristine, etoposide and carboplatin) while group 2 undergoing adjunctive focal consolidative therapy (cryotherapy for anterior tumors and diode laser for posterior lesions) in addition to intravenous chemotherapy.

Patients underwent examination under general anesthesia before the commencement of treatment whereby location and size of the lesions (basal diameter and height) and presence and extent of vitreous or sub retinal seedings and sub-retinal fluid were documented. MR imaging was done to exclude extra ocular extension and trilateral retinoblastoma.

Patients underwent subsequent examination under anesthesia 3 weeks after every cycle. Final size of tumor (basal diameter and height) was documented 3 weeks after the last cycle of chemotherapy.

\section{RESULTS}

Out of the 88 retinoblastoma patients seen in Outpatient clinic, 64 eyes of 43 (48.8\%) patients (24 males (55.8\%)) of Group C and D (40 eyes with group $D(62.5 \%)$ ), aged between 3 months and 6 years (Mean 2 years 3 months) having no previous treatment were randomly divided into two groups. $27(62.8 \%)$ patients had bilateral disease.

The mean basal diameter of the lesions in group 1 reduced from $19.6 \mathrm{~mm}$ (range $13-22 \mathrm{~mm}$ ) to $15.5 \mathrm{~mm}$ (range $12-19 \mathrm{~mm}$ ) with a mean reduction of $4.1 \mathrm{~mm}$ ( $21 \%$ reduction) while the mean basal height of lesion decreased from $3 \mathrm{~mm}$ (2$5 \mathrm{~mm}$ ) to $2.5 \mathrm{~mm}$ (range $1.7-3.5 \mathrm{~mm}$ ) with a mean reduction of $0.5 \mathrm{~mm}$ (16\% reduction) at the end of the 6 cycles of chemotherapy. In group 2, the mean basal diameter of the lesion reduced from $19.2 \mathrm{~mm}$ (range $13-22 \mathrm{~mm}$ ) to $7.3 \mathrm{~mm}$ (range of 12$19 \mathrm{~mm})$ with a mean reduction of $11.9 \mathrm{~mm}(62 \%$ reduction) while the mean basal height reduced from $3 \mathrm{~mm}(2-5 \mathrm{~mm})$ to $1.5 \mathrm{~mm}$ (range 1.3-3.5mm) with a mean reduction of $1.5 \mathrm{~mm}$ ( $50 \%$ reduction) at the end of 6 cycles of chemotherapy and adjuvant focal laser (Table-I).

The mean percentage regression in group1 (height $\times$ base) is $24.5 \%$ while that in group 2 (height $\times$ base) was $59.9 \%$.

The Independent $T$ test value was 36.02 and $p$ value was 0.001 , thus showing that the treatment modality using chemotherapy and local consolidative therapy is more effective than chemotherapy alone in causing regression of retinoblastoma lesions.

\begin{tabular}{|l|c|c|c|}
\hline $\begin{array}{c}\text { Retinoblastoma Treatment } \\
\text { Group }\end{array}$ & $\begin{array}{c}\text { Pre Treatment Basal } \\
\text { Diameter } \mathbf{( m m )}\end{array}$ & $\begin{array}{c}\text { Post Treatment Basal } \\
\text { Diameter (mm) }\end{array}$ & $\begin{array}{c}\text { Mean Reduction } \\
\text { Group 1 (chemo alone) }\end{array}$ \\
\hline Group 2 (chemo and laser) & $19.6(13-22)$ & $15.5(12-19)$ & $4.1(21 \%)$ \\
\hline & $19.2(13-22)$ & $7.3(12-19)$ & $11.9(62 \%)$ \\
\hline
\end{tabular}




\section{DISCUSSION}

In the last century, conservative treatment of retinoblastoma has gained popularity due to adoption of a multidisciplinary approach. The liaison between an ophthalmologist, oncologist and radiotherapist has led to effective treatment guidelines. These are targeted not only for a better overall survival of the patient but also aim to prevent organ loss.

Chemotherapy which was started for retinoblastoma in 1990s caused significant tumor reduction. Later, it was combined with external beam radiotherapy (EBRT) to gain added benefit. ${ }^{12}$ This combination was superior to enucleation as a globe salvage procedure. External Beam Radiotherapy is still largely used in developing countries including Pakistan as reported in literature ${ }^{8-11}$ but has been downgraded in the western world due to its complications. ${ }^{1}$

This combined modality of chemotherapy and adjunctive focal laser has been investigated in the past all over the world. Chemotherapy reduced tumour volume and focal lasers were applied around it to further destroy tumor cells. It is also believed that lasers disrupt blood ocular barrier and facilitate penetration of chemotherapy to vitreous. This helps to reduce vitreous seeds which are otherwise resistant to systemic chemotherapy. ${ }^{13}$ In 1996, Shields CL \& co workers published results of their research reported 35\% reduction in basal diameter and $49 \%$ reduction in thickness after 2 years of chemotherapy and focal lasers. ${ }^{14}$ Another study in 1996, Gallili and his co workers showed the benefits of adding cyclosporine to vincristrine and etopside and following laser therapy can effectively control vitreous seeds and $88 \%$ patients were relapse free in these worst prognosis cases. This pilot study further suggested need for RCT to actually know efficacy of various treatment modalities. In 1996, Murphee showed $100 \%$ success of chemotherapy and focal lasers in treating group 1 to 4 and absolute failure in treating group5 by same modality. ${ }^{15}$ However, patients included in these studies belonged to all groups and were classified according to Reese Eleseworth classification. All these studies showed combined modality is more effective than single modality and made it first choice in moderate risk cases.

Studies completed by Indian authors include review article on genetic perspective of disease and various treatment modalities used in treatment of retinoblastoma. ${ }^{16-18}$

No clinical trial is reported in literature in Pakistan comparing these two modalities. Khurram D in his research has included patients treated by combinedmodalitytoobutno comparisonbetween various treatment options has been done. ${ }^{11}$ This index study has proved benefit of adding local consolidative therapy to chemotherapy to treat moderate risk retinoblastoma patients.

In our study we compared the use of chemotherapy alone and chemotherapy plus local consolidative therapy to treat retinoblastoma lesions. This study not only provided a qualitative assessment but also actually showed quantitative superiority of combined treatment with chemotherapy plus local consolidative therapy over chemotherapy alone. We found that combined modality caused $60 \%$ regression of retinoblastoma lesion after six cycles as compared to $24.5 \%$ for chemotherapy.

In past, patients were usually treated with chemotherapy and failure of chemotherapy resulted in worsening of the disease and eventually ending up with enucleation of the eye. This saved the life of patient but organ loss had a huge negative impact on patient's psychology. Some other chemotherapy failure cases had to undergo external beam radiation therapy, hence at risk of secondary malignancies. Adding focal lasers to chemotherapy also helps to avoid long term systemic complications of chemotherapy like renal failure or liver damage.

In our part of the world, there is a late presentation, and the International guidelines fall short of application in our situation. However, better eye of bilateral retinoblastoma patients can be salvaged using chemotherapy and local consolidative therapy.

A limitation in our study was the short duration of 
study period. The final patient outcome in terms of disease control can thus not be documented. Another limitations was that vitreous seeds and subretinal fluid was not properly addressed in statistical analysis. Multifocal lesions were not taken seperately as they are less responsive to therapy than unifocal lesions. Any new tumour growth during treatment period was also not addressed.

There is a need for a multi-centred clinical trial to see the efficacy of various treatment options in the developing world. This will help us formulate our own guideline for the treatment of retinoblastoma as disease presentation and overall mortality is different here.

\section{CONCLUSION}

Chemotherapy combined with local consolidative therapy is more effective in treating retinoblastoma as compared to chemotherapy. This will not only save the life of patient but also help prevent organ loss. This study has laid the foundation stone of comparative analysis for retinoblastoma treatment in our country. This will help us formulate practice guidelines for retinoblastoma treatment in the developing world.

Comparison of reduction in basal diameter post treatment in retinoblastoma Lesions with single modality (chemotherapy) vs Combined Modality (chemotherapy + local consolidative Therapy.

Copyright(C) 13 Apr, 2020.

\section{REFERENCES}

1. Canadian Retinoblastoma Society. National Retinoblastoma strategy Canadian guidelines for care: Stratégie thérapeutique du rétinoblastome guide cliniquecanadien: Can J Ophthalmol. 2009; 44 Suppl2:S1-88.

2. Chaudry S, Sajoo MS, Reddy MA: Update In the management of retinoblastoma. Pak $\mathrm{J}$ Ophthalmol. 2014; 30(4):185-6.

3. Shields CL, Mashayekhi A, Au AK, Czyz C, Leahey A, Meadows AT, Shield JA. The International Classification of Retinoblastoma predicts chemo reduction success. Ophthalmology. 2006 Dec; 113 (12):2276-80.
4. MacCarthy A, Birch JM, Draper GJ, Hungerford JL, Kingston JE, Kroll ME, et al. Retinoblastoma: treatment and survival in Great Britain 1963 to 2002. Br J Ophthalmol. 2009 Jan; 93(1):38-9.

5. Temming P, Lohmann D, Bornfeld N, SauerweinW, Goericke S, Eggert A. Current concepts for diagnosis and treatment of retinoblastoma in Germany: Aiming for safe tumor control and vision preservation. Klin Padiatr. 2012 Oct; 224(6):339-47.

6. Bowman R, Mafwiri M, Luthert P, Luande J, Wood M. Outcome of retinoblastoma in east Africa. Pediatr Blood Cancer. 2008 Jan; 50(1):160-2.

7. Kivelä T. 200 years of success initiated by James Wardrop's 1809 monograph on retinoblastoma. Acta Ophthalmol. 2009 Nov; 87(8):810-2.

8. Bhurgri $\mathrm{Y}$, Muzaffar S, Ahmed R, Ahmed N, Bhurgri H, Usman A, et al Retinoblastoma in Karachi, Pakistan. Asian Pac J Cancer Prev. 2004 Apr-Jun; 5(2):159-63.

9. Bukhari S, Aziz-ur-Rehman BI, Qidwai U. Presentation pattern of retinoblastoma. Pak J Ophthalmol. 2011; $27(3): 142-5$.

10. Islam F, Zafar SN, Siddiqui SN, Khan A. Clinical course of retinoblastoma. JCPSP. 2013 Aug; 23(8):566-9.

11. Khurram D, Zaheer N, Sharif N. Effects of primary chemotherapy, radiotherapy plus local treatments on regression patterns of posterior pole retinoblastoma. Pak J Ophthalmol. 2011; 27(4):214-9.

12. Kingston JE, Hungerford JL, Madreperla SA, Plowman PN. Results of combined chemotherapy and radiotherapy for advanced intraocular retinoblastoma. Arch Ophthalmol. 1996 Nov; 114:1339-43.

13. Munier. FL. Classification and Management of Seeds in Retinoblastoma. Ellsworth Lecture Ghent August 24th 2013. Ophthalmic Genet.2014 Dec; 35(4):193-207.

14. Shields CL, DePotter $P$, Himmelstein B, Shield JA, Meadows AT, Maris JM. Chemo reduction in the initial management of intraocular retinoblastoma. Arch Ophthalmol 1996 Nov; 114: 1330-1338.

15. Murphree AL, Villablanca JG, Deegan WF 3rd, Sato JK, Malogolowkin M, Fisher Aet al. Chemotherapy plus local treatment in the management of intraocular retinoblastoma. Arch Ophthalmol.1996Nov; 114:134856.

16. Kaliki S, Sheilds CL. Retinoblastoma: Achieving new standards with methods of chemotherapy. Indian $\mathrm{J}$ Ophthalmol. 2015 Feb; 63(2): 103-9 
17. Jagadeesan $M$, Khetan V, Mallipatna A. Genetic perspective of retinoblastoma: From present to future. Indian J Ophthalmol. 2016 May; 64(5):332-6.
18. Chawla B, Jain A, and Azad R. Conservative treatment modalities in retinoblastoma. Indian $\mathrm{J}$ Ophthalmol. 2013 Sep; 61(9):479-85.

\begin{tabular}{|c|c|c|c|}
\hline \multicolumn{4}{|c|}{ AUTHORSHIP AND CONTRIBUTION DECLARATION } \\
\hline Sr. \# & Author(s) Full Name & Contribution to the paper & Author(s) Signature \\
\hline 1 & Asma Mushtaq & 1st Author & \\
\hline 2 & Asad Aslam Khan & 2nd Author & \\
\hline 3 & M. Ali Ayaz Sadiq & 3rd Author & An \\
\hline 4 & Seema Qayyum & 4th Author & \\
\hline 5 & M. Rizwan Khan & 5th Author & Whezian \\
\hline 6 & Ahmed Raza & 6th Author & 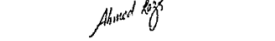 \\
\hline
\end{tabular}

